\title{
TAXA DE CIRCULAÇÃO E PREVISÃO DO COMPORTAMENTO METAL/ESCÓRIA EM MODELO FÍSICO DE UM REATOR RH $^{*}$
}

\author{
Johne Jesus Mol Peixoto ${ }^{1}$ \\ Natália Barros Barony ${ }^{2}$ \\ Weslei Viana Gabriel ${ }^{1}$ \\ Carlos Antonio da Silva ${ }^{3}$ \\ Itavahn Alves da Silva ${ }^{4}$ \\ Varadarajan Seshadri ${ }^{5}$
}

\section{Resumo}

No reator $\mathrm{RH}$, a otimização do fluxo recirculatório de aço entre a câmara de vácuo e a panela é a chave para promover as reações de refino. Neste trabalho, foram utilizados água e solução de cloreto de zinco $\left(\mathrm{ZnCl}_{2}\right)$ como fluido similar ao aço para investigar a taxa de circulação no modelo a frio de um reator $\mathrm{RH}$ em escala 1:7,5. A taxa de circulação foi determinada utilizando o método de pontes de Strain Gages. A técnica PIV foi utilizada de modo a calibrar e validar os resultados. O aumento da vazão de gás de $30 \mathrm{l} / \mathrm{min}$ para $120 \mathrm{l} / \mathrm{min}$ resultou na elevação da taxa de circulação para ambos os fluidos. Cálculos preliminares demonstraram que há grande diferença nas condições de arraste de escória entre os sistemas água/óleo e $\mathrm{ZnCl}_{2} /$ óleo

Palavras-chave: Modelamento físico; PIV; Desgaseificador $\mathrm{RH}$.

\section{CIRCULATION RATE AND PREDICTION OF THE METAL/SLAG BEHAVIOR IN PHYSICAL MODEL OF A RH REACTOR}

\section{Abstract}

In $\mathrm{RH}$ reactor, the optimization of the steel circulatory flow between the vacuum chamber and the ladle is the key factor to promote the refining reactions. In this work, water and zinc chloride solution $\left(\mathrm{ZnCl}_{2}\right)$ were employed as similar fluid to steel in order to investigate the circulation rate in a cold model of a $\mathrm{RH}$ reactor built on 1:7.5 scale. The circulation rate was determined using the method of strain gage bridges. The PIV technique was used to calibrate and validate the results. With an increasing on gas flow rate from $30 \mathrm{l} / \mathrm{min}$ to $120 \mathrm{l} / \mathrm{min}$, the recirculation flow rate increased for both fluids (água e $\mathrm{ZnCl}_{2}$ ). Initial calculations showed that there is a great difference in the conditions of slag dragging between water/oil and $\mathrm{ZnCl}_{2} /$ oil systems.

Keywords: Physical modelling; PIV; RH degasser.

1 Engenheiro Metalúrgico, Msc., Universidade Federal de Ouro Preto (UFOP), Ouro Preto, Minas Gerais, Brasil.

2 Graduanda em Engenharia Metalúrgica, Escola de Minas, Universidade Federal de Ouro Preto (UFOP), Ouro Preto, Minas Gerais, Brasil.

3 Engenheiro Metalúrgico, Ph.D., Professor, DEMET, Escola de Minas, Universidade Federal de Ouro Preto (UFOP), Ouro Preto, Minas Gerais, Brasil.

4 Engenheiro Metalúrgico, DSc., Professor, DEMET, Escola de Minas, Universidade Federal de Ouro Preto (UFOP), Ouro Preto, MG, Brasil.

5 Engenheiro Metalúrgico, Dr. Ing., Professor Emérito, Universidade Federal de Minas Gerais (UFMG), Belo Horizonte, Minas Gerais, Brasil. 


\section{INTRODUÇÃO}

O processo de desgaseificação no reator $\mathrm{RH}$ (Ruhrstal Heraeus) tem sido largamente aplicado no refino secundário do aço, objetivando-se descarburação, remoção de nitrogênio, hidrogênio e inclusões, adição de elementos de liga, entre outros. Neste processo, o refino se baseia na interação entre aço líquido e fase gasosa. O grau de vácuo, a taxa de circulação bem como a agitação tem efeitos importantes nas taxas de reação (ZHANG et al. [1]). Como a medição da taxa de circulação na indústria é difícil de ser realizada, Park et al. [2] destacam a importância da utilização de modelos físicos e simulações matemáticas para investigar os efeitos de diversos parâmetros sobre a taxa de recirculação.

Yang et al. [3] lembram que a etapa de refino do aço no $\mathrm{RH}$ representa uma das últimas oportunidades para realizar a dessulfuração, e por este motivo, o reator $\mathrm{RH}$ tem sido aplicado na produção de aços ultrabaixo teor de enxofre. Para simular a injeção de escória dessulfurante, estes pesquisadores injetaram óleo (uma mistura de dois tipos de óleo para obter $\rho=661 \mathrm{~kg} / \mathrm{m}^{3}$, de modo a obedecer 0 critério de similaridade adotado) na câmara de vácuo de um modelo físico de um reator $\mathrm{RH}$ (escala 1:2,8). Analisando a evolução da camada de óleo no topo da panela, eles avaliaram o tempo de residência e a quantidade de óleo disperso no interior do líquido em função da vazão de gás para determinar a melhor configuração para dessulfuração. Costa et al. [4] propôs um processo de dessulfuração no $\mathrm{RH}$ pela adição de uma camada de escória sobrenadante no interior da câmara de vácuo após a subida do aço sob efeito da depressão. Foi utilizado um modelo matemático aplicado a condições industriais para analisar a dessulfuração do aço e avaliar a influência de parâmetros como composição química e volume de escória.

Deve-se destacar que nestes e em outros trabalhos que abordam a dessulfuração do aço no $\mathrm{RH}$, não se investigou as condições para o arraste da escória adicionada na câmara de vácuo. Além disso, a formação de uma emulsão de escória-metal é de considerável importância prática, pois o aumento da área interfacial causada pela dispersão de uma fase líquida na outra é responsável pelas elevadas taxas de reação que geralmente são encontradas em processos como no LD, no forno panela, entre outros.

No modelamento físico, quando se estuda as interações entre metal e escória, o sistema água/óleo possui uma limitação quanto à diferença de densidade. De modo a modificar este parâmetro, uma prática comum é utilizar soluções aquosas: de cloreto de zinco, como por exemplo, Lin e Guthrie [5] e Arruda [6]; ou cloreto de sódio, Savolainen et al. [7]. Para aplicar uma solução de $\mathrm{ZnCl}_{2}$ como fluido similar ao aço no modelamento físico de um reator $\mathrm{RH}$ é necessário desenvolver um método para determinar a taxa de circulação, pois o método por condutivimetria comumente utilizado para este fim, não pode ser aplicado devido à alta concentração de íons na solução. Neste trabalho, utilizou-se um sistema de medição indireta da taxa de circulação a partir de uma ponte de strain gage. Foram utilizados água e solução de cloreto de zinco $\left(\mathrm{ZnCl}_{2}\right)$ como fluido similar ao aço para investigar o efeito da vazão de gás e imersão das pernas sobre a taxa de circulação no reator $\mathrm{RH}$.

\section{MATERIAIS E MÉTODOS}

Para a realização dos testes de simulação física foi utilizado um modelo do sistema $\mathrm{RH}$ construído em acrílico, obedecendo a um fator de escala $\lambda=7,5$. Dados geométricos e operacionais do reator industrial em que este modelo se baseia são 
mostrados na Tabela 1. Além da água ( $\rho=1,0 \mathrm{~g} / \mathrm{cm}^{3}$ e $\left.\mu=1,0 \mathrm{mPa} . \mathrm{s}\right)$, uma solução de $\mathrm{ZnCl}_{2}\left(\rho=1,22 \mathrm{~g} / \mathrm{cm}^{3}\right.$ e $\mu=15,33 \mathrm{mPa}$.s) foi utilizada como fluido similar ao aço. As vazões de gás (ar comprimido) utilizadas foram de $30 \mathrm{l} / \mathrm{min}$ a $120 \mathrm{l} / \mathrm{min}$ (incremento de $10 \mathrm{l} / \mathrm{min}$ ), a imersão das pernas foi $60 \mathrm{~mm}, 100 \mathrm{~mm}$ e $140 \mathrm{~mm}$ e a altura de líquido na câmara de vácuo foi mantida fixa em $70 \mathrm{~mm}$.

Tabela 1. Descrição das Características do Protótipo e do Modelo do reator RH

\section{CARATERÍSTICAS DO REATOR}

\begin{tabular}{|c|c|c|c|}
\hline \multirow{4}{*}{$\begin{array}{l}\text { Dados } \\
\text { Operacionais }\end{array}$} & Capacidade de Vácuo (mBar) & 0,67 & 984 \\
\hline & Temperatura de Trabalho $\left({ }^{\circ} \mathrm{C}\right)$ & 1560 & 25 \\
\hline & Fluido & Aço & Água \\
\hline & Densidade de Fluido $\left(\mathrm{Kg} / \mathrm{m}^{3}\right)$ & 7000 & 1000 \\
\hline \multirow{2}{*}{ Vaso } & Diâmetro Interno (mm) & 2245 & 299,33 \\
\hline & Altura $(\mathrm{mm})$ & 7600 & $65^{*}$ \\
\hline \multirow{4}{*}{ Snorkels } & Comprimento $(\mathrm{mm})$ & 1820 & 242,67 \\
\hline & Diâmetro Interno (mm) & 750 & 100 \\
\hline & Distância entre Linhas de Centro (mm) & 1540 & 205,33 \\
\hline & Profundidade de Imersão $(\mathrm{mm})$ & 450 & 60 \\
\hline \multirow{6}{*}{$\begin{array}{l}\text { Sistema de } \\
\text { Injeção de Gás } \\
\text { de Arraste }\end{array}$} & Gás & Argônio & Ar comp. \\
\hline & Vazão (NL/min) & 2500 & 120 \\
\hline & Densidade de Gás $\left(\mathrm{Kg} / \mathrm{m}^{3}\right)$ & 0,13 & 1,123 \\
\hline & $\begin{array}{l}\text { Pontos de Injeção de gás (Snorkel de } \\
\text { Subida) }\end{array}$ & $\begin{array}{c}16 \text { bicos, } \\
\text { distribuídos em } 2 \\
\text { níveis de injeção }\end{array}$ & $\begin{array}{l}16 \text { bicos, } 2 \\
\text { níveis }\end{array}$ \\
\hline & Distância entre Níveis de Injeção (mm) & 175 & 23,33 \\
\hline & Diâmetro do Bico de Injeção $(\mathrm{mm})$ & 8 & 2,4 \\
\hline
\end{tabular}

*Altura necessária somente para elevação da água, devido o vácuo induzido.

Os parâmetros do modelo presentes na última coluna da Tabela 1 foram determinados utilizando-se a razão de escala $\lambda=7,5$ e os critérios de similaridade adequados, provenientes dos grupos adimensionais e independentes entre si resultantes da aplicação do Teorema de Buckigham sobre as variáveis relevantes ao processo $\mathrm{RH}$.O adimensional de Reynolds não foi tomado em consideração nestes cálculos, pois na perna de subida o fluxo é bifásico e turbulento. Então a viscosidade molecular (que reflete a influência da força viscosa) não é o parâmetro adequado para refletir o fluxo. Os cálculos que resultaram nos dados da Tabela 1 assumem que os parâmetros relevantes ao movimento de líquido na perna de subida são: a força de inércia do líquido; a força peso do líquido presente na perna; a fração da perna de subida ocupada pelo gás; e a força de inércia do gás no ponto de injeção. Note-se que na Tabela 1 estão ausentes as características do vaso inferior. Para este modelo a panela foi substituída por uma caixa retangular, permitindo a eliminação do efeito de curvatura e a realização de análises quantitativas do fluxo do fluido através da técnica PIV. Um desenho esquemático do modelo está apresentado na Figura 1(a) e uma imagem do modelo em acrílico na figura 1 (b).

Além do mais, por questão de conveniência, os bicos de injeção podem não obedecer à escala adotada para o restante do sistema. Esta última particularidade requer adaptar estes critérios, de modo a refletir o sentido do número de Froude modificado (Equação 1):

$$
F r=\frac{V^{2}}{g D} \quad ; \quad N_{V a}=\frac{Q}{D^{2} V} \quad ; \quad F r_{m}=\frac{\rho_{g} Q\left(Q / \varphi^{2}\right)}{\rho_{l} D^{3} g}
$$

Onde: $\rho_{\mathrm{l}}$ e $\rho_{\mathrm{g}}$ é a massa específica do líquido e do gás; $\mathrm{D}$ é o diâmetro da perna de subida; $v$ é a velocidade do líquido; $Q$ é a vazão de gás; $\varphi$ é o diâmetro do bico de injeção e g é a aceleração da gravidade. 

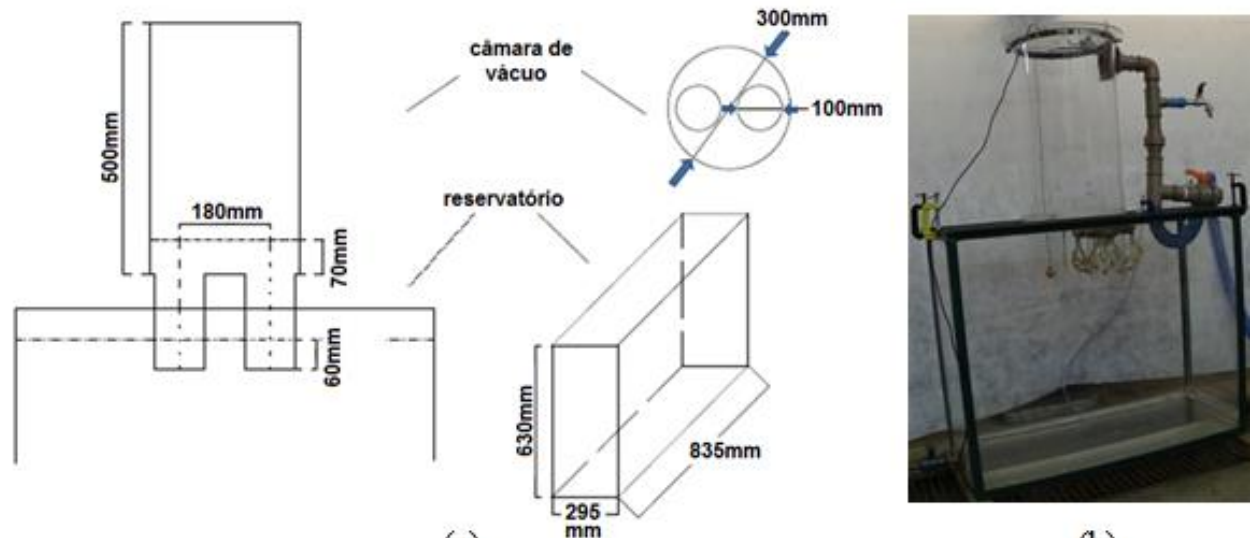

(a)

(b)

Figura 1. (a) Representação esquemática dos parâmetros geométricos do modelo físico utilizado para os experimentos; (b) Modelo de acrílico do desgasseificador RH em escala de 1:7,5.

\subsection{Determinação da Taxa de Circulação - Método de Ponte de Strain Gage (MPSG)}

A determinação da taxa de circulação no modelo de $\mathrm{RH}$, foi utilizado um sistema de medição indireta a partir de uma ponte de strain gage. Parte do esquema experimental para medição da taxa de circulação é mostrado na Figura 2. Uma esfera de massa $m$ e raio $R$ conhecidos é dependurada na extremidade de uma lâmina flexível. A esfera é posicionada na perna de descida, estando portanto sujeita à interação com o fluido que retorna à panela. Os esforços aplicados sobre a lâmina são tais que a mesma se encontra no regime elástico de flexão. Dois Strain Gages são colados na face superior da lâmina e outros dois na face inferior, interconectados de forma a compor uma ponte completa de Wheatstone. Assim, o sinal elétrico da ponte de Wheatstone depende da deformação.

A esfera posicionada no interior da perna de descida está sujeita a esforços correspondentes a o peso, empuxo e força de arraste proporcionada pelo fluido. Maior a vazão de fluido maior a força de arraste, maior a deformação da lâmina e maior o sinal gerado pela ponte. Portanto o sinal da ponte pode ser utilizado para se estimar a vazão (taxa de circulação), como se descreve a seguir.

As deformações sofridas pelas Strain Gages são recebidas numa placa de aquisição de dados $\mathrm{A} / \mathrm{D}$ (analógico/digital) e são fornecidas indiretamente na forma de uma ddp (Volts) gerada pelo condicionador de sinal. São realizadas três séries de leituras por experimento. Primeira leitura, da ponte sem deformação (ponto Zero); sujeita ao peso próprio da lâmina, sem o acoplamento da esfera. Segunda leitura, o sinal da ponte após o acoplamento da esfera, mas no ar, sem a passagem de líquido. Este segundo sinal subtraído do primeiro corresponde ao peso da esfera, o qual é conhecido pela pesagem da mesma em balança de precisão. Portanto estes dois primeiros sinais fornecem a calibração do aparato. A terceira leitura é feita com a circulação do fluido e permite determinar, portanto, a força de arraste (FA). Desta forma:

$$
\begin{gathered}
\text { Resultante }[N]=\left(3^{a} \text { Leitura }-1^{a} \text { Leitura }\right)[V] x \frac{\text { Peso da Esfera }[N]}{\left(2^{a} \text { Leitura-1 } 1^{a} \text { Leitura }\right)[V]} \\
\text { Resultante }[N]=(\text { Peso da Esfera }- \text { Empuxo })+f \frac{1}{2} \rho_{L} V^{2} \pi R^{2}
\end{gathered}
$$

Sabe-se que o fator de friç̧ão f é dado em função do número de Reynolds: 


$$
\begin{gathered}
R e=\frac{\rho_{L} V R}{\mu} \\
f=24 / \mathrm{Re} ; \text { se Reynolds } \leq 1 \\
f=\frac{18,5}{R e^{0,6}} ; \text { se } 1<\text { Reynolds }<1000 \\
f=0,44 ; \text { se Reynolds }>1000
\end{gathered}
$$

Desta forma a Resultante é medida pela ponte de strain gages; peso e empuxo são conhecidos; a força de arraste pode ser calculada:

$$
F A=\text { Resultante }[N]-(\text { Peso da Esfera }- \text { Empuxo })=f \frac{1}{2} \rho_{L} V^{2} \pi R^{2}
$$

Resolve-se então, para determinar o valor de velocidade $\mathrm{V}$, o sistema de equações 4, 5, 6,7 e 8. Daí, a vazão do líquido, ou Taxa de Circulação, pode ser calculada como:

$$
\text { Taxa de Circulação }[\mathrm{Kg} / \mathrm{s}]=\rho_{l}\left(V \frac{\pi D^{2}}{4}\right)
$$

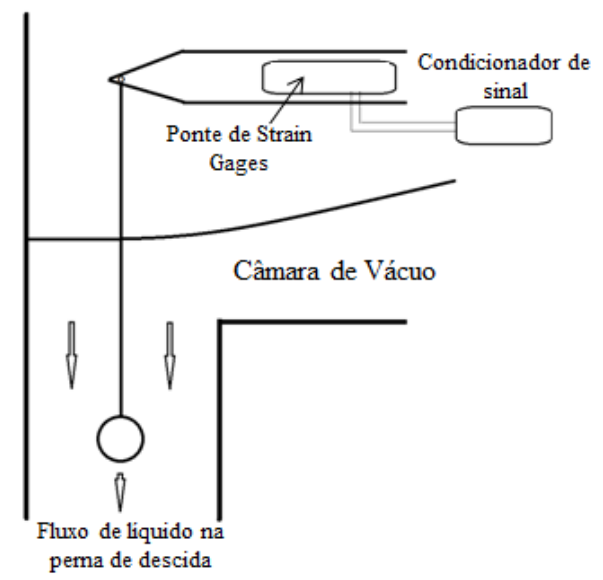

Figura 2. Diagrama esquemático do sistema de medição de taxa de circulação.

Dois pontos precisam ser ressaltados, relativos à aplicação desta metodologia. Para valores de vazão de gás próximos e acima de $90 \mathrm{l} / \mathrm{min}$ nota-se a presença significativa de bolhas de gás no Snorkel de descida, isto é o fluxo deixa de ser monofásico. Além disso, o fluxo na perna de descida se mostra extremamente turbulento, com correntes de recirculação (redemoinhos), que dificultam a aplicação da condição de fluxo unidirecional e simétrico ao redor do corpo esférico. Assim, é necessário utilizar um segundo método para determinação da taxa de circulação, de modo a calibrar e validar os resultados do MPSG.

\subsection{Velocimetria PIV (Particle Image Velocimetry)}

Para calcular a taxa de circulaçãooutro método utilizado foi técnica de velocimetria PIV, pela qual determina-se o perfil de velocidades ao longo da perna de descida, em alturas pré-selecionadas. Para cada combinação de vazão e imersão, foram realizados três aquisições de imagens, sendo adquiridas 100 imagens com frequência de captura de $10 \mathrm{~Hz}$. Considerando uma distribuição radial de velocidades ao longo de linhas coincidentes com o diâmetro da perna de descida, em alturas pré- 
selecionadas, o perfil de velocidades foi integrado de modo a resultar a velocidade média (e então a vazão):

$$
\bar{V}=\int_{o}^{R} \frac{2 \pi r V(r) d r}{\pi R^{2}}
$$

Onde: $\mathrm{R}$ é o raio da perna de descida; $v$ é a velocidade no ponto $r$ determinada pela técnica PIV; e r é a distância do ponto até o centro da perna de descida.

Os valores de vazão (taxa de circulação) obtidos pelas duas técnicas foram comparados, para fins de calibração do método da ponte de strain gages. Esta calibração adicional se faz necessária porque o fluxo na perna de descida se mostra extremamente turbulento, o que dificulta a aplicação da condição de fluxo unidirecional e simétrico ao redor do corpo esférico.Por outro lado quando a vazão de gás aumenta uma fração do mesmo é arrastada até a perna de descida, dificultando as medições via PIV, como mostrado na Figura 3.

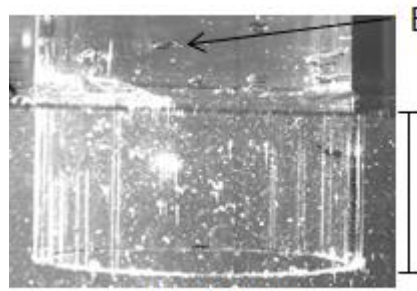

(a) $90 \mathrm{l} / \mathrm{min}$

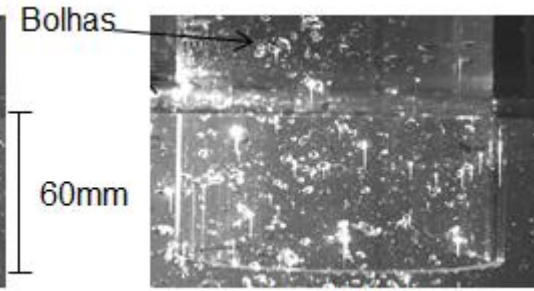

(b) $110 \mathrm{l} / \mathrm{min}$

Figura 3. Presença de bolhas na perna de descida para vazão de gás: (a) 901/min e (b) 1101/min.

\section{RESULTADOS E DISCUSSÃO}

\subsection{Taxa de circulação}

Os perfis de velocidade obtidos via velocimetria PIV para diferentes vazões de gás são mostrados na Figura 4.(a) e 4.(b). A partir destes, três linhas horizontais coincidentes com o diâmetro da perna foram traçadas a $15 \mathrm{~mm}, 30 \mathrm{~mm}$ e $45 \mathrm{~mm}$ acima da saída da perna de descida(Figura 4.(c) e 4.(d)). Nota-se que os valores de velocidade não variam consideravelmente à medida que a linha de medição se afasta da saída da perna de descida (de $15 \mathrm{~mm}$ para $45 \mathrm{~mm}$ ). Deve-se destacar também que para a vazão de $110 \mathrm{l} / \mathrm{min}$ a distribuição de velocidades é completamente assimétrica, devido à presença de grande quantidade de bolhas como foi indicado anteriormente na Figura 3. A partir destes valores determinou-se a vazão de água na perna de descida (taxa de circulação) para cada vazão de gás. 


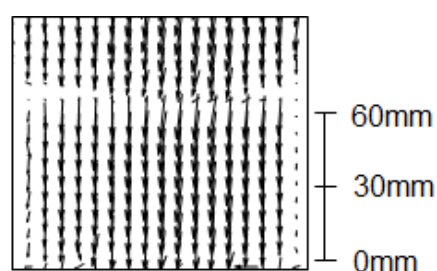

(a) $70 \mathrm{~V} / \mathrm{min}$

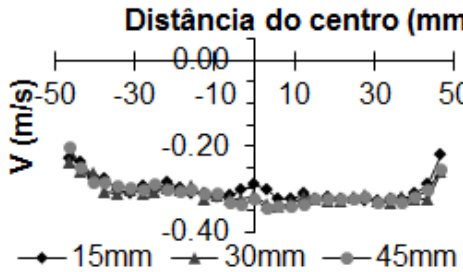

(c) $70 \mathrm{l} / \mathrm{min}$

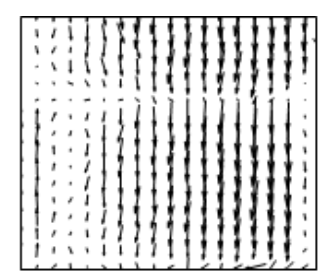

(b) $110 \mathrm{l} / \mathrm{min}$

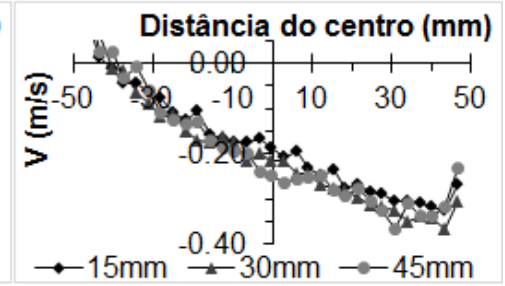

(d) $110 \mathrm{l} / \mathrm{min}$

Figura 4 - Perfil de velocidades na perna de descida obtido via técnica PIV utilizando água para imersão da perna igual a $6 \mathrm{~cm}$ e vazão de gás: (a) 70l/min; (b) 110l/min. Distribuição de velocidade ao longo de uma linha horizontal coincidente com o diâmetro da perna de descida para vazão de gás:(c) $70 \mathrm{l} / \mathrm{min}$ e (d) $110 \mathrm{l} / \mathrm{min}$.

Nas Figuras 5 e 6 tem-se uma comparação entre os valores de taxa de circulação obtidos pelo método da ponte de Strain Gages e pela técnica de velocimetria PIV utilizando água e solução aquosa de $\mathrm{ZnCl}_{2}$, respectivamente. Como é possível observar nestes gráficos, os valores obtidos pelo método da ponte de Strain Gages foram superiores aos obtidos por velocimetria PIV, sendo necessária a utilização de um fator de calibração. Utilizando a ferramenta solver do Excel, tal fator foi determinado por minimização da soma dos quadrados das diferenças entre os dados obtidos via PIV e os valores obtidos pelo MPSG multiplicado pelo fator de calibração. Neste cálculo foram considerados os dados das vazões de $30 \mathrm{l} / \mathrm{min}$ a $901 /$ min dos experimentos utilizando água, devido à presença considerável de bolhas na perna de descida partir da vazão de $90 \mathrm{~L} / \mathrm{min}$ que interferem no cálculo de velocidade via PIV. O fator de calibração encontrado foi 0,6, e este valor é aplicável tanto para a água como para a solução $\mathrm{ZnCl}_{2}$ (Figura 7).

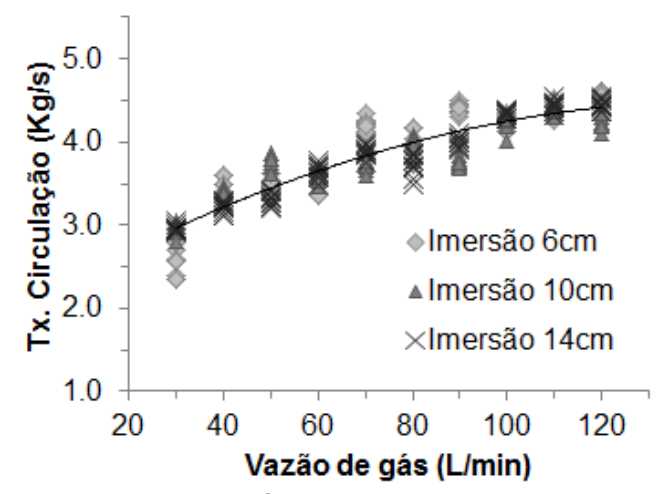

(a) Água - Strain Gage

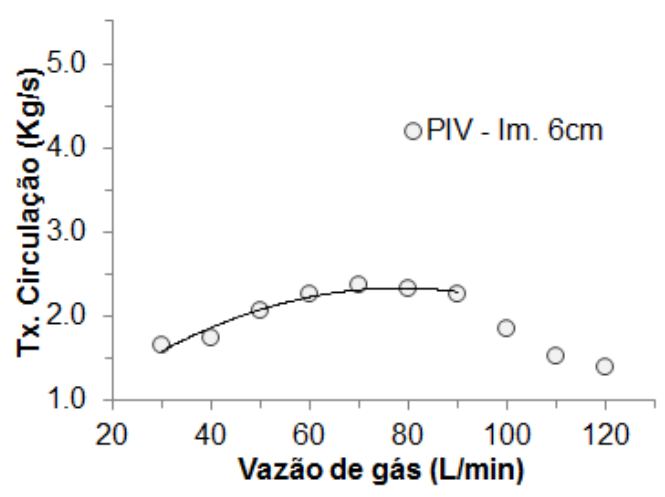

(b) Água - PIV

Figura 52 - Comparação dos valores da taxa de circulação obtidos em experimentos utilizando água: (a) Método da ponte de Strain Gage; (b) técnica PIV.

Na Figura 7 nota-se que a taxa de circulação aumenta com o aumento da vazão de gás injetado, tal como esperado e reportado por Seshadri et al. [8], Almeida et al. [9], Park et al. [2] e outros. Não foi possível identificar a influência da profundidade de imersão da perna sobre a taxa de circulação, tanto para os experimentos utilizando água, quanto solução de $\mathrm{ZnCl}_{2}$. Comparando-se os resultados dos experimentos 
utilizando água (Figura 7(a)) com os valores quando se utiliza a solução de $\mathrm{ZnCl}_{2}$ (Figura 7(b)) observa-se que a taxa de circulação foi ligeiramente maior quanto se utiliza água. Deve-se ressaltar ainda que existe uma tendência à estabilização dos valores de taxa de circulação, para valores maiores de vazão de gás. Park et al. (2000)reportam a existência de um valor de saturação para a vazão de gás, a partir da qual a taxa de circulação tende a decrescer pois o excesso de gás na perna de subida reduz o bombeamento de líquido contido na mesma.

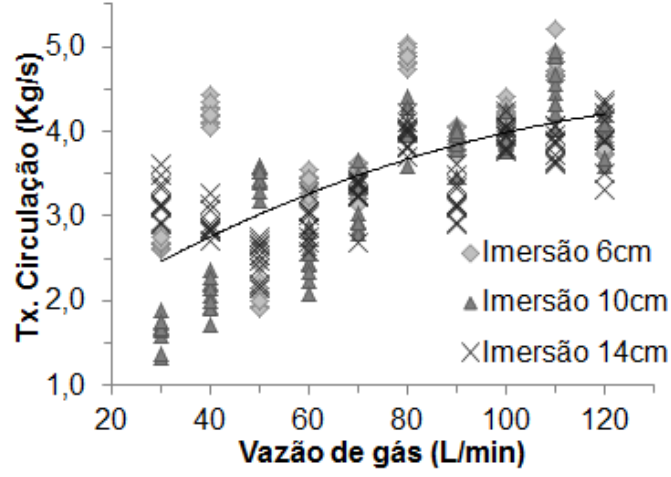

(a) $\mathrm{ZnCl}_{2}$ - Strain Gage

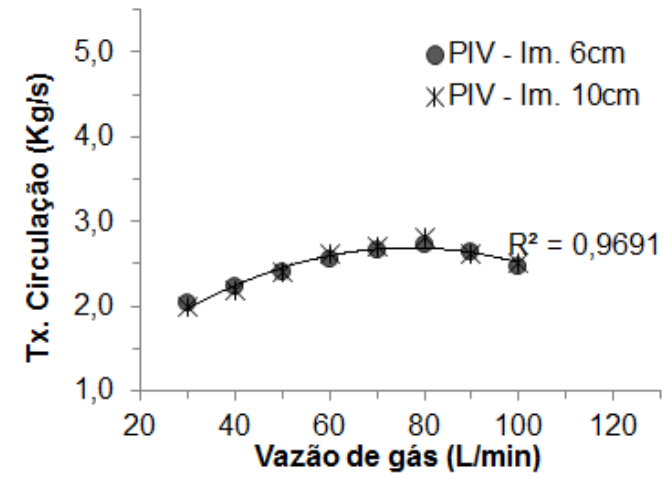

(b) $\mathrm{ZnCl}_{2}$ - PIV

Figura 63 - Comparação dos valores da taxa de circulação obtidos em experimentos solução aquosa de ZnCla:(a) Método da ponte de Strain Gage; (b) técnica PIV.

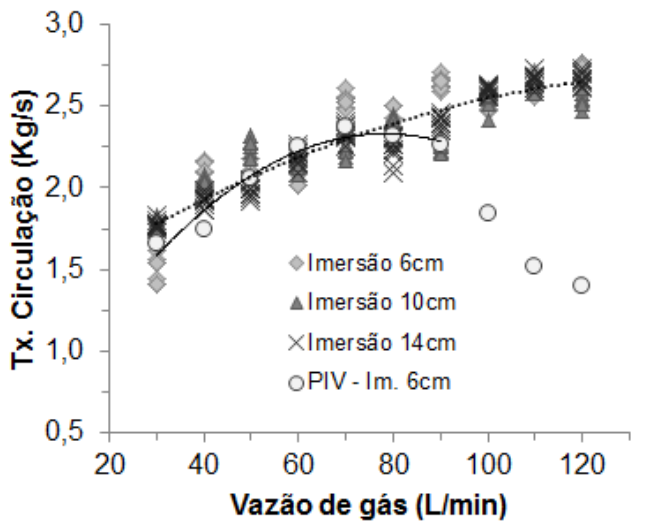

(a) Água

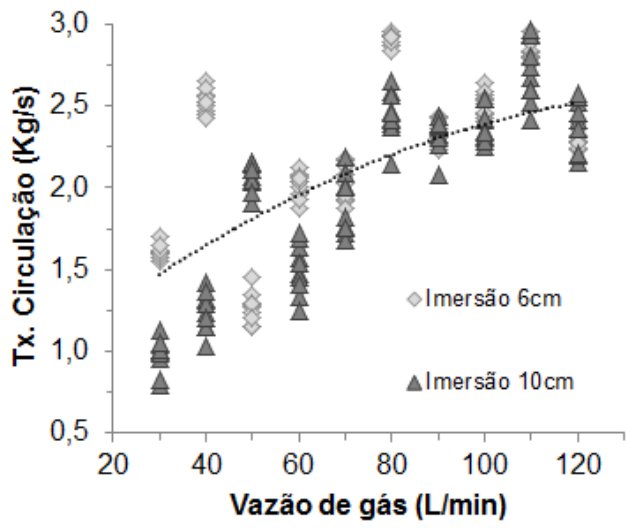

(b) $\mathrm{ZnCl}_{2}$

Figura 74 - Comparação dos valores da Taxa de Circulação obtidos por experimento através do método da ponte de Strain Gage e da técnica PIV com fator de calibração $\alpha=0,6$, utilizando: (a) Água; (b) solução aquosa de $\mathrm{ZnCl}_{2}$.

\subsection{Avaliação do Arraste de escória}

A Tabela 2 apresenta algumas expressões encontradas na literatura para determinação de velocidade crítica (mínima) para ocorrer entranhamento de escória causada pela força de cisalhamento devido à turbulência na interface metal escória, e diâmetro crítico (máximo) da gota entranhada (Hibbeller e Thomas [10]). Considerando a utilização de um óleo de silicone $\left(\rho=0,95 \mathrm{~g} / \mathrm{cm}^{3}, \mu=526 \mathrm{mPa} . \mathrm{s}\right.$,

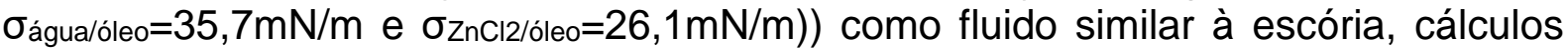
preliminares para analisar as condições de arraste resultam nos valores de $v_{\text {crít }}$ e $d_{c r i ́ t}$ contidos na terceira e quarta colunas da Tabela 2. Observa-se que em função da diferença na densidade, o sistema $\mathrm{ZnCl}_{2} /$ óleo indica velocidade crítica $28 \%$ maior 
que o sistema água/óleo, enquanto o tamanho máximo das gotículas arrastadas é cerca de $65 \%$ menor que água/óleo.

Tabela 2. Cálculos preliminares para determinação das condições de arraste no modelo do reator RH considerando os sistemas $\mathrm{ZnCl}_{2} /$ óleo e água/óleo.

$\begin{array}{llccc}\text { Velocidade Crítica } & \text { Observações } & \begin{array}{c}V_{\mathrm{ZnCl2}} \\ (\mathrm{m} / \mathrm{s})\end{array} & \begin{array}{c}\mathrm{V}_{\text {água }} \\ (\mathrm{m} / \mathrm{s})\end{array} & \begin{array}{c}\mathrm{V}_{\mathrm{ZnC12}} / \mathrm{V}_{\text {água }} \\ (\%)\end{array} \\ \end{array}$

\begin{tabular}{|c|c|c|c|c|}
\hline$\Delta V_{\text {crit }}=\sqrt[4]{4 g\left(\rho_{l}-\rho_{u}\right) \sigma_{u l} \frac{\left(\mu_{l}+\mu_{u}\right)^{4}}{\left(\rho_{u} \mu_{l}^{2}+\rho_{l} \mu_{u}^{2}\right)^{2}}}$ & $\begin{array}{c}\text { Efeito de viscosidade } \\
\text { na teoria de Kelvin- } \\
\text { Helmholtz [11]. }\end{array}$ & 0.120 & 0.092 & $131 \%$ \\
\hline$\Delta V_{c r i t}=\sqrt{8} \cdot \sqrt[4]{\frac{g \sigma_{u l}}{\rho_{l}}(1-r)}$ & $\begin{array}{c}R_{\text {curv. da interface }}<R_{\text {gotículas }} \\
{[12] .}\end{array}$ & 0.233 & 0.183 & $128 \%$ \\
\hline$\Delta V_{c r i t}=\sqrt[4]{48} \cdot \sqrt[4]{\frac{g \sigma_{u l}}{\rho_{l}}\left(\frac{1}{r^{2}}-\frac{1}{r}\right)}$ & $\begin{array}{c}\text { Balanço de energia da } \\
\text { partícula arrastada } \\
\text { [13]. }\end{array}$ & 0.246 & 0.175 & $141 \%$ \\
\hline$\Delta V_{\text {crit }}=\sqrt{12.3} \cdot \sqrt[4]{\frac{g \sigma_{u l}}{\rho_{l}}(1-r)}$ & $\begin{array}{l}\text { Panela com injeção de } \\
\text { gás [14]. }\end{array}$ & 0.328 & 0.233 & $141 \%$ \\
\hline Diâmetro Crítico & Observações & $\begin{array}{l}d_{\mathrm{ZnC12}} \\
(\mathrm{mm})\end{array}$ & $\begin{array}{l}\text { dágua } \\
(\mathrm{mm})\end{array}$ & $\begin{array}{c}d_{\mathrm{znC12} / \mathrm{dágua}} \\
(\%)\end{array}$ \\
\hline$d=\frac{0.534 \cdot \sigma_{u l}^{0.693}}{g^{0.564}\left(\rho_{l}-\rho_{u}\right)^{0.130} \rho_{u}^{0.306}} \frac{\mu_{u}^{0.114}}{\mu_{l}^{0.372}}$ & $\begin{array}{l}\text { Interface água/óleo } \\
\text { [15]. }\end{array}$ & 3.1 & 13.1 & $23 \%$ \\
\hline$d=2 \cdot \sqrt{\frac{\sigma_{u l}}{g\left(\rho_{l}-\rho_{u}\right)}}$ & Ref. [12]. & 6.3 & 17.1 & $37 \%$ \\
\hline$d=\sqrt{12} \cdot \sqrt{\frac{\sigma_{u l}}{g\left(\rho_{l}-\rho_{u}\right)}}$ & Ref. [13]. & 10.9 & 29.6 & $37 \%$ \\
\hline
\end{tabular}

*Onde: g gravidade, $\rho_{l}$ densidade da fase mais densa, $\rho_{u}$ densidade da fase sobrenadante, $\mu_{l}$ viscosidade da fase mais densa, $\mu_{u}$ viscosidade da fase sobrenadante, $\sigma_{u l}$ tensão interfacial entre as fases mais densa e sobrenadante, $r=\frac{\rho_{u}}{\rho_{l}}$.

Da análise da taxa de circulação observa-se que para as vazões de gás de $301 / \mathrm{min}$ a $120 \mathrm{l} / \mathrm{min}$, os valores de velocidade média na perna de descida variam de $0,22 \mathrm{~m} / \mathrm{s}$ a $0,34 \mathrm{~m} / \mathrm{s}$ para a água e $0,15 \mathrm{~m} / \mathrm{s}$ a $0,27 \mathrm{~m} / \mathrm{s}$ para a solução $\mathrm{ZnCl}_{2}$. Considerando que estes valores são iguais a velocidade máxima na interface fluido/óleo no interior da câmara de vácuo, é possível estimar que o arraste de óleo ocorrerá para todas as vazões para o sistema água/óleo enquanto que para $\mathrm{ZnCl}_{2} /$ óleo o arraste ocorrerá apenas para as maiores vazões de gás (acima de $701 / \mathrm{min}$ ). Isto demonstra que conclusões retiradas de experimentos envolvendo água e óleo claramente superestimam o efeito do entranhamento de escória. Esta indicação é importante pois a multiplicação de área interfacial metal escória é apontada como determinante para a velocidade do processo de refino.

\section{CONCLUSÃO}

A taxa de circulação foi determinada utilizando o método de pontes de Strain Gages. A técnica PIV foi utilizada de modo a calibrar e validar os resultados. O aumento da vazão de gás de $30 \mathrm{l} / \mathrm{min}$ para $120 \mathrm{l} / \mathrm{min}$ resultou na elevação da taxa de circulação de $1,5 \mathrm{~kg} / \mathrm{s}$ para $2,75 \mathrm{~kg} / \mathrm{s}$ (para a água) e $1,5 \mathrm{~kg} / \mathrm{s}$ para $2,5 \mathrm{~kg} / \mathrm{s}$ (para a solução de 
$\mathrm{ZnCl}_{2}$ ). Cálculos iniciais demonstraram que há grande diferença nas condições de arraste de escória entre os sistemas água/óleo e $\mathrm{ZnCl}_{2} /$ óleo: o arraste de óleo ocorre em todas as vazões de gás para o sistema água/óleo enquanto que no sistema $\mathrm{ZnCl}_{2} /$ óleo o arraste ocorre apenas para as maiores vazões de gás, sendo que a velocidade crítica foi em média $35 \%$ maior e o diâmetro máximo da gotícula arrastada foi cerca de $65 \%$ menor para o sistema $\mathrm{ZnCl}_{2} /$ óleo. $^{\circ}$

\section{Agradecimentos}

Ao CNPq, à CAPES, à Fundação Gorceix e à FAPEMIG pelo apoio.

\section{REFERÊNCIAS}

1 ZHANG, J.; LIU, L.; ZHAO, X.; LEI, S. \& DONG, Q. Mathematical Model for Decarburization Process in RH Refining Process. ISIJ Int. 2014;54(7):560-1569.

2 PARK, Y-G., YI K-W \& AHN S-B. The Numerical Calculation of Circulation Flow Rate in the Degassing Rheinstahl-Heraeus Process. ISIJ International. 40; 8: 749-755, 2000.

3 YANG, H.; YANG, S.; LI, J. \& ZHANG, J. A Mathematical Model to Characterize RH Desulfurization Process. J. of Iron and Steel Research Int. 2014;21(11):995-1001.

4 COSTA, L.T \& TAVARES, R.P. Dessulfuração do aço em desgaseificador RH por meio de modelo matemático. In: Associação Brasileira de Metalurgia, Materiais e Mineração. Anais do 46은 Seminário de Aciaria - Internacional; 2015; Rio de Janeiro, Brasil. São Paulo: ABM; 2015. P.552-561.

5 LIN Z \& GUTHRIE R.I.L. Modeling of Metallurgical Emulsions. Metallurgical and Materials Transactions B. 1994; 25(B):855-864.

6 ARRUDA J.D. Análise Via Modelagem Física e Matemática da Turbulência na Interface Metal/Escória na Região do Menisco em um Molde de Lingotamento Contínuo de Placas [dissertação de mestrado]. Ouro Preto - REDEMAT, UFOP, 2015.

7 SAVOLANEIN, J, FABRITIUS, T \& MATTILA, O. Effect of Fluid Physical Properties on the Emulsification. ISIJ International. 2009; 49(1):29-36.

8 SECHADRI, V.; SILVA, C. A. da; SILVA, I. A. da; VARGAS, G. A \& LASCOSQUI, P. S. $B$. Decarburisation rates in RH-KTB degasser of CST steel plant through physical modelling study. Ironmaking and Steelmaking. 2006; 33(1):34-38.

9 Almeida, A. T. P., Alvarenga, J. A., Belarmino, T. J. P., CARDOSO, A. C. P., NASCIMENTO, A. A., TAVARES, R. P., et al. Physical Modeling of Vacuum Decarburization in an RH Degasser. AISTech. 2006; 1: 761-770.

10 HIBBELER, L.C \& THOMAS, B.G. Mold Slag Entrainment Mechanisms In Continuous Casting Molds. In: AISTech Proceedings, 2013, Pittsburg, PA, USA, 2013.p.1215-1230.

11 FUNADA, T \& JOSEPH, D.D. Viscous potential flow analysis of kelvin-helmholtz instability in a channel. J. of Fluid Mechanics. 2001;445:263-283. Apud HIBBELER e THOMAS [10].

12 NAKATO, H; SAITO, K; OGUCHI, Y; NAMURA, N \& SORIMACHI, K "Surface quality improvement of continuously cast blooms by optimizing solidification in early stage," in 70th Steelmaking Conference Proceedings, pp. 427-431, The Iron and Steel Society, 1987. Apud HIBBELER, L.C e THOMAS B,G [10].

13 ASAI, S, "Fluid flow and mass transfer in a refining process by use of stirring," in Proceedings of the 100th and 101st Nishiyama Memorial Lecture, The Iron and Steel Institute of Japan, 1984. p.65-100. Apud HIBBELER L.C e THOMAS B.G [10].

14 XIAO, Z; PENG, Y \& LIU, C "Modelling study of the entrapment phenomena at the slagmetal interface in the gas-stirred ladle," Chinese J. of Metal Science and Technology. 1987;3:187-193. Apud SAVOLAINEN et al. [7].

15 HARMAN, J. M \& CRAMB, A.W - "A study of the effect of fluid physical properties upon droplet emulsification," in 79th Steelmaking Conference Proceedings, The Iron and Steel Society. 1996, p.773-784. Apud HIBBELER, L.C \& THOMAS B,G [10]. 